Revista 2020

\title{
El Centro de Escritura Digital como una estrategia pedagógica institucional ${ }^{*}$
}

\author{
Gerzon Yair Calle-Álvareza
}

Resumen: En una sociedad que se comunica y conecta a través del texto, el fortalecer las habilidades de escritura se convierte en un aspecto fundamental en la escuela. Este artículo tiene como propósito identificar los factores que permitieron que un Centro de Escritura Digital (CED) operara como una estrategia institucional para el fortalecimiento de la escritura académica. El método utilizado fue el estudio de casos. Participaron dos instituciones educativas, una pública y otra privada, que ofrecían educación media, de la ciudad de Medellín, Colombia. Se aplicaron cuestionarios con preguntas abiertas a estudiantes, profesores y tutores. En los resultados, se abordaron las categorías de orientación de la escritura académica, servicios integrados a la institución, proyección e identidad institucional, promoción de los servicios e integración curricular. En un CED el uso de las Tecnologías de la Información y la Comunicación (TIC) supera la enseñanza del uso de herramientas, implica el fortalecimiento de los procesos de alfabetización digital desde las áreas y asignaturas del currículo escolar, donde se involucren los directivos, profesores, estudiantes y padres de familia. En las conclusiones se afirma que el reconocimiento del CED como parte de la comunidad educativa genera acciones orientadas al mantenimiento y sostenibilidad, como un componente del proyecto educativo institucional. Además, la integración del CED con la gestión directiva y académica es un factor que aporta a la diferenciación institucional y sostenibilidad de la estrategia en el tiempo.

Palabras clave: centro de recursos; currículum; práctica pedagógica; tecnología educativa.

Recibido: 22 de marzo de 2020 Aceptado: 18 de mayo de 2020

Disponible en línea: 22 de octubre de 2020.

Cómo citar: Calle-Álvarez, G. Y. (2020). El Centro de Escritura Digital como una estrategia pedagógica institucional. Academia y Virtualidad, 13(2), 69-90. https://doi.org/10.18359/ravi.4670

* Artículo derivado de la tesis doctoral titulada "El centro de escritura digital. Una posibilidad para el fortalecimiento de la producción de textos académicos en la Educación Media", en el marco del proyecto de investigación El Centro de escritura digital. Posibilidades y retos para promover la calidad de las prácticas de escritura académica en la escuela primaria (2015-2018), financiado por el Comité para el Desarrollo de la Investigación (CODI) de la Universidad de Antioquia.

a Doctor en Educación. Profesor de la Facultad de Educación de la Universidad de Antioquia, Medellín, Colombia. Correo electrónico: gerzon.calle@udea.edu.co ORCID: https://orcid.org/0000-0002-4083-6051 


\section{The digital Writing Center as an institutional pedagogical strategy}

Abstract: In a society that communicates and connects through text, strengthening writing skills becomes a fundamental aspect in school. The purpose of this article is to identify the factors that allowed a Center for Digital Writing (CED) to operate as an institutional strategy to strengthen the academic writing. The method used was the case study. Two secondary education institutions participated, one public and one private, in the city of Medellín, Colombia. Questionnaires with open questions were applied to students, teachers, and tutors. In the results, the categories of academic writing counseling, institution integrated services, institutional projection and identity, promotion of services and curricular integration were addressed. In a CED, the use of Information and Communication Technologies (ICT) goes beyond the teaching of tools usage, it entails strengthening the digital literacy processes from the areas and subjects of the school curriculum, where management, teachers, students and parents are involved. The conclusions confirm that recognizing the CED as part of the educational community generates actions aimed at its maintenance and sustainability, as a component of the institutional educational project. In addition, the integration of the CED with the directive and academic management is a factor that contributes to the institutional differentiation and sustainability of the strategy over time.

Keywords: Resource Center; resume; pedagogical practice; educational technology.

\section{O Centro de Escrita Digital como uma estratégia pedagógica institucional}

Resumo: Numa sociedade que se comunica e se conecta por meio do texto, o fortalecer das habilidades de escrita torna-se um aspecto fundamental na escola. Este artigo tem como propósito identificar os fatores que permitiram que um Centro de Escrita Digital (ced) operasse como uma estratégia institucional para o fortalecimento da escrita acadêmica. O método utilizado foi o estudo de casos. Participaram duas instituições educativas, uma pública e outra privada, que ofereciam educação média, da cidade de Medellín, Colômbia. Aplicaram-se questionários com perguntas abertas a estudantes, professores e tutores. Nos resultados, abordaram-se as categorias de orientação da escrita acadêmica, serviços integrados à instituição, projeção e identidade institucional, promoção dos serviços e integração curricular. Num ced o uso das Tecnologias da Informação e Comunicação (tic) supera o ensino do uso de ferramentas, implica o fortalecimento dos processos de alfabetização digital nas áreas e matérias do currículo escolar, em que se envolvam os diretores, professores, estudantes e pais de família. Nas conclusões afirma-se que o reconhecimento do ced como parte da comunidade educativa gera ações orientadas à manutenção e sustentabilidade, como um componente do projeto educativo institucional. Além disso, a integração do ced com a gestão diretiva e acadêmica é um fator que contribui para a diferenciação institucional e sustentabilidade da estratégia no tempo.

Palavras-chave: centro de recursos; currículo; prática pedagógica; tecnologia educativa. 


\section{Introducción}

El sistema educativo, en su búsqueda por responder al fortalecimiento de las prácticas de producción de textos en los diferentes niveles que lo integran, ha diseñado estrategias para acercar a los estudiantes a la escritura. En Colombia, la Ley General de Educación (Ley 115, 1994) ha planteado un fin para la educación básica y media relacionado con el desarrollo de habilidades comunicativas. Igualmente, creó una asignatura en Humanidades y Lengua Castellana, la cual está pensada para ser eje articulador de este objetivo. Sin embargo, la escuela viene comprendiendo que la escritura es un tema transversal que no puede estar bajo la responsabilidad de un solo profesor o asignatura.

Desde los abordajes de la escritura en la escuela en el contexto colombiano, se puede retomar los "Estándares Básicos de Competencias en Lenguaje, Matemáticas, Ciencias Sociales, Ciencias Naturales y Competencias Ciudadanas" publicados por el Ministerio de Educación Nacional (MEN, 2006), e identificar que en el área de Lenguaje hay una línea de trabajo relacionada directamente con la producción textual; sin embargo, en las demás áreas se reconocen mecanismos de comunicación que requieren ejercicios de lectura y escritura propios de cada una de las áreas, con propósitos orientados a la comprensión, interpretación, solución de problemas, análisis de situaciones, construcción de ideas. Este enfoque permite que el estudiante adquiera cada uno de los saberes propios de las diferentes áreas y asignaturas del currículo escolar.

Las propuestas de promoción y fortalecimiento de la lectura y escritura en la educación básica y media colombiana son diversas. Entre ellas se encuentran, por ejemplo, el periódico escolar virtual (Ávila, 2009), o los proyectos de lectura digital y biblioteca escolar (Torres, 2013), taller de escritores (Rodríguez, Muñoz y Ángel, 2015) o revista escolar (Aponte y Cruz, 2015). Lo anterior permite evidenciar que las instituciones educativas se vienen preocupando por mejorar los procesos de producción e interpretación en sus estudiantes y que buscan estrategias que respondan a sus realidades.

Eleftheriou (2011) y Utne (2016) afirman que en los últimos años los profesores se han preocupado por desarrollar Centros de Escritura (CE) en instituciones educativas de educación secundaria, media y superior en los Estados Unidos. Los CE como estrategia pedagógica tienen como propósito fortalecer la escritura académica en los estudiantes, y para ello consideran el lugar del estudiante, los profesores, administradores escolares y las dinámicas institucionales en general. Los CE nacieron en la educación superior (Calle-Arango, 2020), pero desde hace más de dos décadas se vienen incoporando en las instituciones de educación secundaria y media.

Con el auge de las Tecnologías de la Información y la Comunicación (TIC), los CE han diversificado las posibilidades de acceso y servicios (Calle-Arango, 2020). Algunos mantienen la connotación de $\mathrm{CE}$, pero se apoyan en recursos digitales para la administración y divulgación de la información; otros operan de manera presencial y virtual; y otros se han consolidado en los espacios virtuales, adquiriendo distintas nominaciones, como Laboratorio de Escritura en Línea, Centro de Escritura Virtual, Centro de Escritura Digital (CED), entre otros.

Carrasco (2019) plantea que si se desea formar lectores y escritores críticos es necesario adaptar el currículo a las necesidades del entorno escolar. Implementar un CED en el contexto colombiano requiere que se hagan las adaptaciones curriculares y administrativas necesarias para que pueda operar como una estrategia institucional y logre perdurar en el tiempo. Un CED esta orientado a promover y fortalecer la escritura académica en los estudiantes; además, aporta a la integración de los procesos de alfabetización digital en el currículo escolar. Esto repercute en la proyección de los estudiantes en la vida social, profesional y laboral. Stueart (2012) señala que el bajo rendimiento en lectura y escritura afecta el éxito estudiantil en la universidad; además, le implica al estudiante invertir tiempo y dinero en un aprendizaje que debió haber adquirido en la educación secundaria y media.

En Colombia, los profesores tienen un promedio de estudiantes por grupo de treinta y cinco o más, lo que dificulta trabajar y acompañar los procesos de escritura con los estudiantes que se 
encuentran bajo su orientación. Utne (2016) plantea que esta situación ha generado que los estudiantes con dificultades en los procesos de producción escrita agudicen sus problemas o se demoren más tiempo en superarlos, y los estudiantes con buenos niveles de escritura no tengan la oportunidad de continuar en esa línea de crecimiento y mejora personal. El CED se diferencia de otras estrategias pedagógicas porque permite que los estudiantes tengan un espacio de atención individual por parte de un tutor, ya para dialogar y superar sus problemas de escritura, ya para fortalecer sus propias prácticas de lectura y escritura académica.

En un CED, la escritura académica es el motivante para que los profesores, estudiantes y padres de familia logren desarrollar estrategias de interacción y participación, enfocados en que los estudiantes potencien sus prácticas de producción de textos. Las relaciones entre el CED y la comunidad educativa superan el trabajo en el aula de clases; se puede acceder a los servicios desde un computador con conexión a internet, si bien se requiere que los usuarios participen de los servicios. Este artículo tiene como propósito presentar los resultados de las posibilidades del CED como estrategia institucional para la promoción y fortalecimiento de la escritura académica en la educación media.

\section{Referentes metodológicos}

La investigación se desarrolló desde el método de estudio de casos. Participaron dos instituciones educativas, una pública y otra privada, que ofrecían educación media, de la ciudad de Medellín, Colombia. Cada institución fue abordada como un caso independiente, sin embargo, en algunos factores se contrastaron los resultados. A partir de una lectura de contexto, a cada institución se le diseñó un CED que integraba servicios de tutoría académica, talleres virtuales de escritura y biblioteca de recursos digitales; desde sus estructuras administrativas y pedagógicas, cada institución educativa operó el CED durante dieciocho meses.

Los CED en la educación media y en el contexto colombiano son una estrategia pedagógica reciente, que no tiene trayectoria investigativa, por lo que la utilización del estudio de casos fue pertinente en la medida que se indagó por un fenómeno educativo contemporáneo (Yin, 1994; Yacuzzi, 2005). En la presente investigación hubo una preocupación por comprender la profundidad de la realidad educativa dentro de las relaciones que se establecieron entre el CED y la comunidad educativa.

Para la recolección de la información se diseñó un cuestionario de indagación final que buscó identificar aspectos que no fueron visibles en los procesos de observación del investigador y que indagaba sobre cómo el CED fue reconocido por diferentes estamentos de la comunidad educativa. Este instrumento se aplicó a los estudiantes, profesores y tutores, en un solo momento y al final de proceso de investigación. García (2002) afirma que un cuestionario es un sistema de preguntas racionales que permite la recolección de información de fuentes primarias; además, permite establecer un puente entre el planteamiento del problema y las respuestas que se obtienen de la población. Para el diseño del cuestionario de indagación final se redactaron una serie de preguntas a partir de las siguientes categorías: diseño y desarrollo del CED y el CED en la institución educativa.

Diseño y desarrollo del CED. Esta categoría tenía como objetivo aportar a la mirada global del proceso de diseño y funcionamiento del CED en las instituciones. Contenía tres preguntas abiertas orientadas a identificar las ventajas y/o desventajas en los procesos de acompañamiento de la escritura académica, la percepción sobre los módulos que lo constituían y otros aspectos que consideraran que no estaban en el CED y hubiera sido bueno haber encontrado.

El CED en la institución educativa. Esta categoría estaba asociada a la comprensión del CED en las dinámicas institucionales. Contenía tres preguntas enfocadas a visibilizar los beneficios o los problemas que este generó en la vida institucional, el reconocimiento de las estrategias de promoción y el uso de los recursos del CED en las actividades institucionales.

Para la aplicación del cuestionario se diseñó un formulario en Google Drive. Vale anotar que, aunque se mantuvieron las mismas categorías iniciales 
para la construcción de las preguntas, estas variaban en su redacción, buscando comprensión por parte de la población objetivo. Para lograr procesos de confiabilidad en la aplicación de los cuestionarios se solicitó a un experto que los revisara y validara. El experto era un doctor en educación, con investigaciones sobre educación, escritura y TIC, y con experiencia en construcción de proyectos transversales para la educación básica y media. El experto consideró que la estructura del instrumento respondía a los intereses del momento en que se encontraba la investigación.

El caso 1 (IE pública), lo constituía una población de 302 estudiantes y 17 profesores y el caso 2 (IE privada), 120 estudiantes y 11 profesores. El investigador seleccionó 61 estudiantes de la IE pública y 17 de la IE privada para que respondieran el instrumento. Los estudiantes seleccionados hacían parte de la educación media y habían asistido a dos o más servicios del CED. A estos estudiantes se les envió un correo invitándolos a responder el cuestionario. Al final, 33 estudiantes de la IE pública y 7 de la IE privada enviaron sus respuestas.

Para los profesores el investigador seleccionó 10 de la IE pública y 5 de la IE privada para que respondieran el instrumento. Los profesores seleccionados tenían bajo su responsabilidad por lo menos un área o asignatura en la educación media, habían accedido a la plataforma del CED y utilizado algunos de los materiales en sus clases; además, estos no eran tutores del CED. A estos profesores se les envió un correo invitándolos a responder el cuestionario. Al final, 6 profesores de la IE pública y 2 de la IE privada enviaron sus respuestas.

Para los tutores del CED, el instrumento fue aplicado, al final de la intervención, a 6 tutores de la IE pública y a 2 de la IE privada. Vale anotar que esos datos corresponden al 100\% de los tutores de cada uno de los casos que se encontraban activos al momento de la aplicación del instrumento.

El proceso de análisis de la información se realizó mediante el método de contenido, el cual permite una comprensión desde lo sintáctico, semántico y pragmático. Para el presente estudio se abordó la información desde lo semántico y pragmático, porque se buscó conocer el significado de las respuestas desde el mensaje y sus relaciones con el contexto, esto permitió lograr profundidad en la comprensión de las dinámicas del CED, desde cada una de las categorías propuestas en los instrumentos. Para la consolidación y análisis de la información se siguieron las indicaciones de Tesch (1990), estableciendo unidades relevantes para el propósito de la investigación.

Posteriormente, se realizó una lectura interpretativa y explicativa de las unidades, desde las categorías planteadas inicialmente y las emergentes. Para ello, se subieron todas las respuestas dadas por los estudiantes, profesores y tutores a una tabla de Microsoft Excell. Luego, se identificaron unidades de análisis desde las respuestas, teniendo presentes las categorías iniciales. Sin embargo, el investigador debía estar atento a las categorías que emergían de la lectura en profundidad de las respuestas. Posteriormente, los datos se ingresaron al programa estadístico sPSs, para determinar el número y porcentaje de respuestas por participante y categoría, el alcance y frecuencia por tamaño de grupo, y generar los gráficos de relaciones.

En el proceso investigativo se trabajó con dos grupos focales dentro de cada comunidad educativa, especialmente con los profesores y los estudiantes, lo que implicó tener presentes unos principios éticos.

La investigación se llevó a cabo después de que el representante legal de cada una de las Instituciones Educativas otorgó la autorización por escrito. En la autorización quedó constancia de que la investigación no pretendía generar prejuicios contra los miembros de la comunidad educativa o la institución educativa, y que siempre se partiría de los principios éticos para el tratamiento de la información, lo que implicó mantener ambas instituciones en el anonimato.

En el consentimiento informado de los profesores y estudiantes (o del representante legal del estudiante) participantes en el desarrollo de la investigación quedó consignado el respeto al buen nombre de los participantes, la autorización del uso de la información con fines investigativos, así como las responsabilidades de las partes en el proceso investigativo. 


\section{Resultados}

Lograr la integración del CED en el Proyecto Educativo Institucional (PEI) es un factor que aporta a la diferenciación y sostenibilidad en el tiempo del CED, en las políticas institucionales. Las instituciones educativas que adopten un CED dentro de sus PEI partirán de sus realidades y políticas internas sobre la lectura y la escritura, o sobre la incorporación de las TIC en las actividades académicas. En ambos casos se tenía una política institucional sobre lectura y escritura, y uso de las TIC en el aula, reflejadas en el horizonte institucional, proyectos institucionales y talento humano competente. Además, en sus inicios el proyecto de diseño e implementación del CED fue dado a conocer y avalado por los Consejos Académicos y Directivos, y en determinados momentos desde los Consejos Académicos se hacía seguimiento al accionar del CED en la institución. A continuación, se presen$\tan$ los resultados de cada uno de los factores que permitieron el reconocimiento del CED como una estrategia pedagógica institucional, en cada una de las instituciones educativas participantes en la investigación.

\section{Orientación de la escritura académica desde las realidades institucionales}

Entre los estudiantes que utilizaron el CED se indagó por sus ventajas para el acompañamiento de la escritura académica apoyada por TIC (ver Tabla 1). En ambos casos, un amplio porcentaje de los estudiantes identificó como ventaja el favorecer el aprendizaje y la construcción del conocimiento desde la escritura (caso 1, 48,4\% y caso 2, 71.4\%), lo que repercutirá en una proyección de la institución, en las participaciones de los estudiantes en eventos de ciudad. El componente didáctico (caso $1,45.2 \%$ y caso $2,57.1 \%$ ) se reconoció desde los recursos para acceder a instrucciones y orientaciones para el fortalecimiento de la escritura académica, sea con una orientación de un tutor, sea de manera autónoma. Además, los estudiantes consideraron que el material didáctico que se encontró disponible en la plataforma de CED era de calidad.

Para cierto porcentaje de estudiantes, el componente técnico fue una ventaja (caso 1, 29\% y caso $2,14.3 \%)$, lo que se explicaba en las rutas de acceso y navegación en la plataforma del CED, estructura modular y organización de la información. Igualmente, algunos estudiantes afirmaron que fortalece sus habilidades de lectura y escritura aplicando las estructuras discursivas de las áreas (caso 1, 16.1\% y caso 2, 42.9\%). Meuller (2006) afirma que un CE brinda herramientas para reforzar y solucionar problemas en aspectos relacionados con la escritura. Finalmente, algunos estudiantes consideraron que el CED permitió el acceso a la información (caso $1,16.1 \%$ y caso 2, 28.6\%), debido a que desde la plataforma se accedía a contenido propio del CED u otras páginas web, que aportaba a la autoformación en diferentes tipologías textuales.

Werner (2013) plantea que los CE se han integrado a los espacios de enseñanza en las instituciones educativas. En el caso 1, los profesores

Tabla 1. Ventajas del CED identificadas por los estudiantes

\begin{tabular}{|c|c|c|c|c|c|c|}
\hline & \multicolumn{2}{|l|}{ Total } & \multicolumn{2}{|l|}{$\begin{array}{l}\text { Caso 1: } \\
\text { IE Pública }\end{array}$} & \multicolumn{2}{|l|}{$\begin{array}{c}\text { Caso 2: } \\
\text { IE Privada }\end{array}$} \\
\hline & $n .^{\circ}$ de respuestas & $\%$ & n. ${ }^{\circ}$ de respuestas & $\%$ & n. ${ }^{\circ}$ de respuestas & $\%$ \\
\hline Favorece el aprendizaje y el conocimiento & 20 & 52,6 & 15 & 48,4 & 5 & 71,4 \\
\hline Componente didáctico & 18 & 47,4 & 14 & 45,2 & 4 & 57,1 \\
\hline Componente técnico & 10 & 26,3 & 9 & 29,0 & 1 & 14,3 \\
\hline Fortalece la lectura y escritura & 8 & 21,1 & 5 & 16,1 & 3 & 42,9 \\
\hline Acceso a información & 7 & 18,4 & 5 & 16,1 & 2 & 28,6 \\
\hline
\end{tabular}

Fuente: elaboración propia. 
identificaron seis aciertos y en el caso 2, dos aciertos, los cuales coincidieron con el caso 1 (ver Tabla 2). En ambos casos, un amplio porcentaje de profesores reconoció el apoyo del CED a la enseñanza desde las áreas (caso 1, 66.7\% y caso 2, 50\%), en el sentido de permitir ampliar los espacios de consulta y las herramientas para la elaboración de ayudas didácticas específicas. Además, en el caso 1, el $50 \%$ de los profesores reconoció el material didáctico que se encuentra en el CED para la enseñanza y aprendizaje de la escritura. En ambos casos se consideró un acierto las posibilidades que brindó el CED para el aprendizaje autónomo del estudiante (caso $1,33.3 \%$ y caso $2,50 \%$ ), donde este tenía la posibilidad de seleccionar el material que considerara pertinente para el desarrollo de su tarea de escritura, como un apoyo extraescolar.

En el caso 1, el 50\% de los profesores identificó como un acierto el aprendizaje colaborativo, los estudiantes podían aprender del otro y con el otro, se crearon momentos de diálogos, discusiones e intercambio de ideas. Stueart (2012) afirma que un CE para la educación media facilita el aprendizaje colaborativo y fomenta el liderazgo creativo en los estudiantes. Un ejemplo de ello fueron los foros de discusión en los talleres virtuales de escritura. También se identificó que el uso de las TIC en el aula puede ser difícil (caso 1, 33.3\%), pero el CED favoreció el acercamiento de las TIC para la enseñanza desde las áreas. La estructura técnica del CED soportada en Moodle se consideró un acierto (caso $1,16.7 \%)$, debido a que permitió que los recursos fueran descargados y aplicados en las actividades de aula, con un soporte básico de las Tic.

Por otra parte, algunos estudiantes de ambos casos identificaron como una desventaja las dificultades en la navegación e interacción en la plataforma del CED (caso 1, 25.8\% y caso 2, 14.3\%), reflejadas en la ubicación de las ventanas, la lentitud del sistema y la incomprensión de los íconos para publicar comentarios (ver Tabla 3). Es una situación que desde el comienzo se consideró, pero como no era comprensible que en la portada del CED apareciera todo el contenido, fue necesario diseñar rutas de navegación. Villasevil (2016) afirma que el sistema de navegación debe ser claro para que el usuario pueda identificar las rutas de trabajo. El sistema lento se asociaba a las condiciones institucionales de capacidad de conexión a internet, para soportar la proyección de videos simultáneamente, en varios equipos de cómputo en la institución educativa. Estas desventajas generan retos institucionales como la formación de los estudiantes en rutas de navegación web y lectura de íconos, además de la ampliación del ancho de banda de internet. En el caso 1, el 9.7\% de los estudiantes expresaron dificultad en la comprensión de las temáticas planteadas en los talleres virtuales de escritura.

En el caso 1, cinco de los profesores identificaron cuatro desaciertos en el proceso de implementación del CED (ver Tabla 4). Faltaron mecanismos de difusión de los servicios que permitieran que más profesores y estudiantes se incorporaran a las

Tabla 2. Aciertos del CED identificados por los profesores

\begin{tabular}{|c|c|c|c|c|c|c|}
\hline & \multicolumn{2}{|l|}{ Total } & \multicolumn{2}{|l|}{$\begin{array}{l}\text { Caso 1: } \\
\text { IE Pública }\end{array}$} & \multicolumn{2}{|l|}{$\begin{array}{l}\text { Caso 2: } \\
\text { IE Privada }\end{array}$} \\
\hline & n. ${ }^{\circ}$ de respuestas & $\%$ & n. ${ }^{\circ}$ de respuestas & $\%$ & n. ${ }^{\circ}$ de respuestas & $\%$ \\
\hline Apoyo a la enseñanza desde las áreas & 5 & 62,5 & 4 & 66,7 & 1 & 50,0 \\
\hline Material didáctico & 3 & 37,5 & 3 & 50,0 & 0 & 0,0 \\
\hline Aprendizaje colaborativo & 3 & 37,5 & 3 & 50,0 & 0 & 0,0 \\
\hline Autonomía & 3 & 37,5 & 2 & 33,3 & 1 & 50,0 \\
\hline Incorporación de las TIC a la enseñanza & 2 & 25,0 & 2 & 33,3 & 0 & 0,0 \\
\hline Estructura técnica & 1 & 12,5 & 1 & 16,7 & 0 & 0,0 \\
\hline
\end{tabular}

Fuente: elaboración propia. 
Tabla 3. Desventajas del ced identificadas por los estudiantes

\begin{tabular}{|c|c|c|c|c|c|c|}
\hline & \multicolumn{2}{|l|}{ Total } & \multicolumn{2}{|l|}{$\begin{array}{l}\text { Caso 1: } \\
\text { IE Pública }\end{array}$} & \multicolumn{2}{|l|}{$\begin{array}{l}\text { Caso 2: } \\
\text { IE Privada }\end{array}$} \\
\hline & n. ${ }^{\circ}$ de respuestas & $\%$ & n. ${ }^{\circ}$ de respuestas & $\%$ & n. ${ }^{\circ}$ de respuestas & $\%$ \\
\hline Dificultades en la navegación e interacción & 9 & 23,7 & 8 & 25,8 & 1 & 14,3 \\
\hline Dificultad temática & 3 & 7,9 & 3 & 9,7 & 0 & 0,0 \\
\hline
\end{tabular}

Fuente: elaboración propia.

Tabla 4. Desaciertos del CED identificados por los profesores

\begin{tabular}{|c|c|c|c|c|c|c|}
\hline & \multicolumn{2}{|l|}{ Total } & \multicolumn{2}{|l|}{$\begin{array}{l}\text { Caso 1: } \\
\text { IE Pública }\end{array}$} & \multicolumn{2}{|l|}{$\begin{array}{l}\text { Caso 2: } \\
\text { IE Privada }\end{array}$} \\
\hline & n..$^{\circ}$ de respuestas & $\%$ & $n .^{\circ}$ de respuestas & $\%$ & n. ${ }^{\circ}$ de respuestas & $\%$ \\
\hline $\begin{array}{l}\text { Faltan mecanismos de difusión de los } \\
\text { servicios }\end{array}$ & 2 & 25,0 & 2 & 33,3 & 0 & 0,0 \\
\hline $\begin{array}{l}\text { Recursos tecnológicos por parte de la } \\
\text { institución }\end{array}$ & 2 & 25,0 & 2 & 33,3 & 0 & 0,0 \\
\hline $\begin{array}{l}\text { Dificultades de aplicación por parte de } \\
\text { algunos docentes }\end{array}$ & 1 & 12,5 & 1 & 16,7 & 0 & 0,0 \\
\hline Permiso de administrador limitante & 1 & 12,5 & 1 & 16,7 & 0 & 0,0 \\
\hline
\end{tabular}

Fuente: elaboración propia.

actividades convocadas por el CED (caso 1, 33.3\%). Los recursos tecnológicos de la institución educativa pública fueron insuficientes para atender las actividades escolares y los servicios del CED (caso 1, 33.3\%). Algunos profesores tuvieron dificultades en la incorporación de las actividades del CED en el trabajo de aula, debido a que no dominaban las herramientas tecnológicas para ello (caso 1, 16.7\%). Finalmente, un profesor consideró que los permisos de administrador limitan las posibilidades para la creación de nuevos contenidos (caso 1, $16.7 \%$ ), debido a que fue una función asociada a un equipo técnico que se encontraba fuera de la institución educativa.

El análisis de las relaciones entre las ventajas y desventajas identificadas por los estudiantes permitió comprobar que los procesos de acompañamiento desde el CED tuvieron mayor influencia en el favorecimiento del aprendizaje y el conocimiento desde las diferentes áreas. Lo anterior concuerda con la postura de Simpson (1991) y Griswold (2003) sobre la importancia de los CE en el aprendizaje de los estudiantes. Por otra parte, las dificultades en la navegación e interacción en la plataforma del CED y los problemas en el abordaje de las temáticas por parte de los estudiantes se debieron al acceso de la información que se requería para los propósitos por los cuales participaban de los servicios del CED.

En el caso 1, el 33.3\% de los tutores ${ }^{1}$ consideró que el ejercicio de escritura más complejo de acompañar fue el taller de la reseña, debido a que era una actividad grupal, lo que hacía que fuera dificultoso resolver las dudas de cada estudiante. El $16.7 \%$ de los tutores dijo que el más complicado fue el taller de la escritura de presentaciones; aunque los estudiantes están familiarizados con la tecnología, fue complejo lograr que utilizaran las herramientas para el trabajo educativo, también se les dificultó concretar sus ideas de forma escrita.

En el caso 1, el 16.7\% de los tutores expresó que el ejercicio más complejo de orientar fue la escritura de un mapa conceptual, durante una tutoría

1 Si bien los tutores del CED podían ser profesores de la institución educativa, desde el CED eran los responsables de orientar los servicios. 
académica, debido a los preconceptos del estudiante, que tenía ideas erradas sobre cómo construir un mapa conceptual. En el caso 2, el 50\% de los tutores respondieron que el informe de laboratorio, en una tutoría académica, fue complejo de acompañar por el uso del lenguaje técnico de las Ciencias Naturales. Calle-Álvarez (2019) considera la tutoría como una oportunidad de conversación e intercambio de ideas sobre la producción textual del estudiante.

En el caso 1, el 33.3\% de los tutores consideró que la producción textual mejor lograda por un estudiante después del servicio fue la escritura de presentaciones, por las características multimodales de este tipo de textos. El 33.3\% de los tutores afirmó que fue la autobiografía, debido a que al realizar un trabajo personalizado se orientaba el resultado, logrando identificar los errores y corregirlos en trabajo conjunto con el estudiante. También fue productivo que el estudiante tuviera varias tutorías.

En el caso 1, el 16.7\% de los tutores expresó que el escrito mejor logrado fue el proyecto de investigación porque hubo motivación por parte de los estudiantes para aceptar las observaciones realizadas y mejorarla; además, el proceso de realimentación del tutor fue significativo. El 16.7\% de los tutores dijo que fue la carta, por los sentimientos y emociones del estudiante en el proceso de producción. En el caso 2, el 50\% de los tutores manifestaron que el escrito mejor logrado fue el cuento, por el interés del estudiante para participar en un concurso nacional de este género.

Los estudiantes, profesores y tutores reconocieron que el CED aportó una potencialización de las dinámicas de la escritura académica en tres niveles: actividades de clases, propuestas institucionales y eventos de ciudad y país. Se identifica que los componentes didáctico y técnicos permitieron que estudiantes y profesores hicieran uso de los servicios y recursos para mejorar sus producciones escriturales, lo que repercutía en mejores resultados, lo que era valorado por los profesores en las áreas. Sin embargo, el uso de los recursos tecnológicos para la enseñanza y aprendizaje sigue siendo un aspecto por mejorar por parte de los profesores y estudiantes.

\section{Servicios que se integren a la institución educativa}

En ambos casos de estudio, los estudiantes expresaron que las estrategias de tutoría académica, talleres virtuales de escritura y biblioteca virtual del CED aportaron a los procesos de aprendizaje de la escritura académica (ver Tabla 5). Las razones que expusieron señalaron, en gran medida, la aplicabilidad en las actividades académicas para el aprendizaje (caso 1, 41.9\% y caso 2, 57.1\%), pues reconocían que los módulos propuestos tenían relación con lo que se desarrollaba dentro de las áreas. Por otra parte, valoran las orientaciones didácticas que encuentran en los módulos del CED (caso 1, 9.7\% y caso 2, 42.9\%), desde las posibilidades de leer y aplicar una guía en la tarea de escritura hasta la de participar de un taller virtual. Finalmente, un mínimo porcentaje de estudiantes en ambos casos identificó como positivo el diseño visual y facilidad de uso (caso $1,6.5 \%$ y caso 2, 14.3\%), lo que proporcionó el ingreso y utilización de los recursos.

Tabla 5. Percepciones de los módulos del CED desde los estudiantes

\begin{tabular}{|c|c|c|c|c|c|c|}
\hline & \multicolumn{2}{|l|}{ Total } & \multicolumn{2}{|l|}{$\begin{array}{l}\text { Caso 1: } \\
\text { IE Pública }\end{array}$} & \multicolumn{2}{|l|}{$\begin{array}{l}\text { Caso 2: } \\
\text { IE Privada }\end{array}$} \\
\hline & n. ${ }^{\circ}$ de respuestas & $\%$ & $n .^{\circ}$ de respuestas & $\%$ & n. ${ }^{\circ}$ de respuestas & $\%$ \\
\hline Aplicabilidad académica/aprendizaje & 17 & 44,7 & 13 & 41,9 & 4 & 57,1 \\
\hline Orientaciones didácticas & 6 & 15,8 & 3 & 9,7 & 3 & 42,9 \\
\hline Diseño visual y facilidad de uso & 3 & 7,9 & 2 & 6,5 & 1 & 14,3 \\
\hline
\end{tabular}

Fuente: elaboración propia. 
Un amplio porcentaje de profesores de ambos casos consideraron que los módulos con que cuenta el CED aportaron a la escritura académica dentro de las áreas que orientan (caso 1, 33.3\% y caso 2, 100\%), justificando que las prácticas de lectura y escritura son parte transversal del currículo escolar (ver Tabla 6). Además, los profesores de Lengua Castellana reconocieron que las temáticas propuestas en los recursos del CED están relacionadas con los planes de áreas de las instituciones educativas, para la educación media.

En el caso 1, el 50\% de profesores manifestó que los módulos que más aportaron al área fueron la biblioteca de recursos virtuales y los talleres virtuales de escritura porque eran un apoyo para la elaboración de textos, entre los cuales se encontró la escritura de una presentación, que eran los más usados en áreas como Tecnología e Informática, Ciencias Naturales, Ciencias Sociales, y además eran complemento al desarrollo del plan de área y al logro de las competencias específicas en los estudiantes.

Varios estudiantes en ambos casos manifestaron que les hubiera gustado encontrar en el CED material de otros temas sobre asuntos de sociedad y juventud (caso 1, 16.1\% y caso 2, 28.6\%), lo que sería pertinente incluir como material para la promoción de la lectura (ver Tabla 7). En el caso 1 , los estudiantes expresaron que les hubiera gustado haber encontrado juegos virtuales (32.3\%) y audiolibros (3.2\%); los primeros son una posibilidad para incorporar en la biblioteca de recursos, donde estos juegos virtuales promuevan las prácticas de lectura y escritura; igualmente, los audiolibros requieren una selección que aporte al canon institucional. También esperaban encontrar guías técnicas para el manejo de programas computacionales (6.5\%), lo que no sería viable, porque desdibujaría los intereses del CED.

Tabla 6. Módulos del CED que aportan a las áreas, profesores

\begin{tabular}{|c|c|c|c|c|c|c|}
\hline & \multicolumn{2}{|l|}{ Total } & \multicolumn{2}{|l|}{$\begin{array}{l}\text { Caso 1: } \\
\text { IE Pública }\end{array}$} & \multicolumn{2}{|l|}{$\begin{array}{l}\text { Caso 2: } \\
\text { IE Privada }\end{array}$} \\
\hline & n. ${ }^{\circ}$ de respuestas & $\%$ & $n .^{\circ}$ de respuestas & $\%$ & n. ${ }^{\circ}$ de respuestas & $\%$ \\
\hline Todos & 4 & 50,0 & 2 & 33,3 & 2 & 100,0 \\
\hline Talleres & 3 & 37,5 & 3 & 50,0 & 0 & 0,0 \\
\hline Biblioteca & 3 & 37,5 & 3 & 50,0 & 0 & 0,0 \\
\hline
\end{tabular}

Fuente: elaboración propia.

Tabla 7. Lo que les hubiera gustado encontrar en el CED a los estudiantes

\begin{tabular}{|c|c|c|c|c|c|c|}
\hline & \multicolumn{2}{|l|}{ Total } & \multicolumn{2}{|l|}{$\begin{array}{l}\text { Caso 1: } \\
\text { IE Pública }\end{array}$} & \multicolumn{2}{|l|}{$\begin{array}{c}\text { Caso 2: } \\
\text { IE Privada }\end{array}$} \\
\hline & n..$^{\circ}$ de respuestas & $\%$ & n. ${ }^{\circ}$ de respuestas & $\%$ & $\mathrm{n} .^{\circ}$ de respuestas & $\%$ \\
\hline Juegos virtuales & 10 & 26,3 & 10 & 32,3 & 0 & 0,0 \\
\hline Nada & 9 & 23,7 & 6 & 19,4 & 3 & 42,9 \\
\hline Material de otros temas & 7 & 18,4 & 5 & 16,1 & 2 & 28,6 \\
\hline Guías técnicas & 2 & 5,3 & 2 & 6,5 & 0 & 0,0 \\
\hline Audiolibros & 1 & 2,6 & 1 & 3,2 & 0 & 0,0 \\
\hline Test de inteligencia & 1 & 2,6 & 1 & 3,2 & 0 & 0,0 \\
\hline Módulo de libre acceso & 1 & 2,6 & 1 & 3,2 & 0 & 0,0 \\
\hline Actividades en inglés & 1 & 2,6 & 0 & 0,0 & 1 & 14,3 \\
\hline
\end{tabular}

Fuente: elaboración propia. 
El 3.2\% de los estudiantes del caso 1 expresó que le hubiera gustado tener la posibilidad de acceder a uno de los módulos del CED de manera libre, sin necesidad de identificarse; ambos CED, sin embargo, estaban circunscritos a las dinámicas institucionales, por lo tanto, desde el comienzo del diseño se determinó que los usuarios serían los estudiantes y profesores, esto con el objetivo de responder a los intereses de esta población. Tener un módulo abierto superaría la capacidad de respuesta institucional. Por otra parte, esperaban encontrar test de inteligencias (caso 1, 3.2\%); es una posibilidad para ser evaluada, debido a que se tendría que hacer una selección que permita a estudiantes y profesores tomar decisiones para el trabajo de la escritura en el aula o de forma individual. En el caso 2 , el $14.3 \%$ de los estudiantes manifestó que le hubiera gustado encontrar material de inglés, sin embargo, desde el inicio del montaje del CED fue una decisión que fuera para el fortalecimiento de la lengua castellana; esto no impide que las instituciones puedan decidir sobre las posibilidades de respuesta de ampliar a una segunda lengua.

El 33.3\% de los profesores del caso 1 expresó que le hubiera gustado encontrar en el CED material de lectura propio de cada área, es decir, textos que circulan en las disciplinas para la formación en comprensión de lectura, lo cual serviría como estrategia para vincular a los estudiantes a la lectura desde la convicción y el gusto (ver Tabla 8). También se expresó el deseo de contar con el CED como un espacio para la libre publicación de contenido desarrollado por los propios los docentes (caso 1, 16.7\%); sobre este particular, desde las orientaciones del CED se determinó que los profesores podían publicar sus propios recursos para la enseñanza, pero debían respetar los principios del CED, además de pasar por un proceso de asesoría y revisión.

Varios profesores esperaban encontrar guías/ talleres de escritura literaria (caso 1, 16.7\%). De hecho, desde la biblioteca de recursos virtuales los estudiantes y profesores tenían acceso a material didáctico para la escritura literaria. Igualmente, les hubiera gustado encontrar guías para la escritura de mapas conceptuales (caso 1, 16.7\%). Los talleres de escritura literaria y las guías de mapas conceptuales son oportunidades de mejora para el CED, que las instituciones pueden incorporar.

En el caso 1, el 50\% de los tutores identificó como fortalezas, en el desarrollo de tutorías académicas y talleres, el componente didáctico del CED, expresado en las posibilidades para los estudiantes de afianzar la escritura como aspecto fundamental y transversal para cualquier área, además, porque actualiza y aporta a los procesos de enseñanza orientados por los profesores (ver Tabla 9). El 33.3\% de los tutores consideró como fortaleza el aporte del CED a la promoción y potenciación de la escritura académica en los estudiantes de la educación media, debido a los servicios y recursos virtuales con que cuenta. En el caso 1, el 16.7\% y en el caso 2, el 50\% de los tutores expresaron que una ventaja del CED es la posibilidad de aprendizaje autónomo en los estudiantes, debido a que los servicios y recursos fueron claros y adecuados para el trabajo de los estudiantes; además, se brindaron indicaciones y pasos específicos para el apoyo en la producción de los textos.

Tabla 8. Lo que les hubiera gustado haber encontrado en el CED a los profesores

\begin{tabular}{|c|c|c|c|c|c|c|}
\hline & Total & & $\begin{array}{c}\text { Caso 1: } \\
\text { IE Pública }\end{array}$ & & $\begin{array}{l}\text { Caso 2: } \\
\text { IE Privada }\end{array}$ & \\
\hline & n..$^{\circ}$ de respuestas & $\%$ & n..$^{\circ}$ de respuestas & $\%$ & n..$^{\circ}$ de respuestas & $\%$ \\
\hline Material de lectura propios de las áreas & 2 & 25,0 & 2 & 33,3 & 0 & 0,0 \\
\hline Sección de libre publicación para docentes & 1 & 12,5 & 1 & 16,7 & 0 & 0,0 \\
\hline Guía mapa conceptuales & 1 & 12,5 & 1 & 16,7 & 0 & 0,0 \\
\hline Guías / Talleres escritura literaria & 1 & 12,5 & 1 & 16,7 & 0 & 0,0 \\
\hline
\end{tabular}

Fuente: elaboración propia. 
Tabla 9. Fortalezas del CED identificadas por los tutores

\begin{tabular}{|c|c|c|c|c|c|c|}
\hline & \multicolumn{2}{|l|}{ Total } & \multicolumn{2}{|l|}{$\begin{array}{l}\text { Caso 1: } \\
\text { IE Pública }\end{array}$} & \multicolumn{2}{|l|}{$\begin{array}{l}\text { Caso 2: } \\
\text { IE Privada }\end{array}$} \\
\hline & n. ${ }^{\circ}$ de respuestas & $\%$ & n. ${ }^{\circ}$ de respuestas & $\%$ & n. ${ }^{\circ}$ de respuestas & $\%$ \\
\hline Componente didáctico & 3 & 37,5 & 3 & 50,0 & 0 & 0,0 \\
\hline Fortalecer la escritura académica & 2 & 25,0 & 2 & 33,3 & 0 & 0,0 \\
\hline Autonomía para el estudiante & 2 & 25,0 & 1 & 16,7 & 1 & 50,0 \\
\hline
\end{tabular}

Fuente: elaboración propia.

En el caso 1, el 33.3\% y en el caso 2, el 50\% de los tutores reconocieron como una dificultad las limitaciones de ingreso a la plataforma, debido a que los estudiantes tienen una contraseña y usuarios asignados, pero al momento del servicio no la recordaban, lo que implicó procesos de recuperación, y en ocasiones fue más complejo porque los estudiantes no recordaban el correo con el cual fueron matriculados al CED. En el caso 1, el 16.7\% de los tutores expresó que hubo dificultades en los saberes previos de los estudiantes, para afrontar los talleres virtuales de escritura, asociados al uso de los discursos propios de las áreas. En el caso 2, el 50\% de los tutores consideró que la institución no contaba con suficiente personal para atender la demanda del CeD (ver Tabla 10).

A partir de las relaciones entre las fortalezas y dificultades que plantearon los tutores de ambos casos se identificó que los problemas de saberes previos en los estudiantes generan obstáculos al momento de lograr el aprendizaje autónomo que se propone en algunos de los recursos del CED. Sin embargo, se desconoce que los estudiantes no necesariamente deben tener un saber específico, que desde la práctica se logran niveles de aprendizaje autónomo. Igualmente, el componente didáctico del CED es un aporte al fortalecimiento de la escritura académica en los estudiantes de la educación media.

\section{Aportes a la proyección e identidad institucional}

Faigley (1998) plantea que los CE deben asumir un papel de liderazgo en las instituciones educativas. Un amplio porcentaje de estudiantes en el caso 1, y uno más reducido del caso 2 (caso 1, 51.6\% y caso $2,28.6 \%)$, reconocieron que el CED aportó a la institución educativa un fortalecimiento en el aprendizaje de la lectura y escritura, reflejados en acciones como el reconocimiento de las tipologías textuales, la incorporación de referencias bibliográficas a los textos, o la criticidad al momento de incorporar recursos multimodales a los escritos (ver Tabla 11). Igualmente, la promoción de la escritura académica al interior de las áreas (caso 1, 29\% y caso 2, 42.9\%) visibilizó la importancia del dominio de los discursos propios de cada área. Además, en algunos casos se evidenció el compromiso de los estudiantes para el desarrollo de las actividades de escritura acompañadas desde el CED (caso 1, 6.5\% y caso 2, 28.6\%).

Tabla 10. Debilidades del CED identificadas por los tutores

\begin{tabular}{|c|c|c|c|c|c|c|}
\hline & \multicolumn{2}{|l|}{ Total } & \multicolumn{2}{|l|}{$\begin{array}{c}\text { Caso 1: } \\
\text { IE Pública }\end{array}$} & \multicolumn{2}{|l|}{$\begin{array}{l}\text { Caso 2: } \\
\text { IE Privada }\end{array}$} \\
\hline & $n .^{\circ}$ de respuestas & $\%$ & $n .^{\circ}$ de respuestas & $\%$ & n. ${ }^{\circ}$ de respuestas & $\%$ \\
\hline Limitante para ingresar & 3 & 37,5 & 2 & 33,3 & 1 & 50,0 \\
\hline Tutores insuficientes para cubrir demanda & 1 & 12,5 & 0 & 0,0 & 1 & 50,0 \\
\hline Saberes previos de estudiantes & 1 & 12,5 & 1 & 16,7 & 0 & 0,0 \\
\hline
\end{tabular}

Fuente: elaboración propia. 
El 6.5\% de los estudiantes del caso 1 identificó la plataforma como un aporte del CED a la institución; esta se reconoce como un espacio virtual propio, que es de uso de los estudiantes, profesores y padres de familia, donde pueden ingresar y hacer uso de los recursos alojados. Holtz (2014) plantea que el uso de recursos digitales en el CE aporta al cumplimiento de los objetivos del mismo. Finalmente, el 3.2\% de los estudiantes del caso 1 consideró que el CED aporta a la proyección institucional en el barrio y la ciudad, como un aspecto para mostrar en diversos espacios académicos.

El 50\% de los profesores del caso 1 consideró que uno de los beneficios del CED es haber permitido la interacción entre los integrantes de la institución educativa, así, estudiantes de diferentes grupos intercambian ideas y generan discusiones alrededor de sus propios textos (ver Tabla 12). Igualmente, el 33.3\% valoró el apoyo virtual para la escritura con que contó la institución, ya que las actividades convocadas por el CED no se limitaban a los tiempos escolares; los estudiantes y profesores participaron de los servicios del CED, en tiempo extraescolar y desde sus casas. Eckard y Stroudsburg (2013) y Holtz (2014) valoran la incorporación de las herramientas digitales en la gestión de los CE, para la atención de los usuarios, como un elemento que favorece los tiempos y los espacios de acceso.

También se generó integración de áreas, aprendizaje transversal (caso 1,50\%). Por un lado, esto se observa en la evaluación desde diferentes áreas, por ejemplo, en el caso 1, donde los textos que producían los estudiantes eran abordados y evaluados por profesores de las áreas de Tecnología e Informática, Ciencias Naturales y Educación Física. Por otro lado, los docentes que usaron la plataforma se beneficiaron porque ampliaban sus posibilidades para generar estrategias de aprendizaje.

Tabla 11. Beneficios institucionales del CED identificados por los estudiantes

\begin{tabular}{|c|c|c|c|c|c|c|}
\hline & \multicolumn{2}{|l|}{ Total } & \multicolumn{2}{|l|}{$\begin{array}{l}\text { Caso 1: } \\
\text { IE Pública }\end{array}$} & \multicolumn{2}{|l|}{$\begin{array}{l}\text { Caso 2: } \\
\text { IE Privada }\end{array}$} \\
\hline & $n .^{\circ}$ de respuestas & $\%$ & $n .^{\circ}$ de respuestas & $\%$ & $\mathbf{n} .^{\circ}$ de respuestas & $\%$ \\
\hline Aprendizaje de los estudiantes & 18 & 47,4 & 16 & 51,6 & 2 & 28,6 \\
\hline Promoción escritura académica & 12 & 31,6 & 9 & 29,0 & 3 & 42,9 \\
\hline Compromiso de los estudiantes & 4 & 10,5 & 2 & 6,5 & 2 & 28,6 \\
\hline Plataforma para la institución & 2 & 5,3 & 2 & 6,5 & 0 & 0,0 \\
\hline Proyección institucional & 1 & 2,6 & 1 & 3,2 & 0 & 0,0 \\
\hline
\end{tabular}

Fuente: elaboración propia.

Tabla 12. Beneficios institucionales del CED identificados por los profesores

\begin{tabular}{|c|c|c|c|c|c|c|}
\hline & \multicolumn{2}{|l|}{ Total } & \multicolumn{2}{|l|}{$\begin{array}{c}\text { Caso 1: } \\
\text { IE Pública }\end{array}$} & \multicolumn{2}{|l|}{$\begin{array}{l}\text { Caso 2: } \\
\text { IE Privada }\end{array}$} \\
\hline & $n .^{\circ}$ de respuestas & $\%$ & n. ${ }^{\circ}$ de respuestas & $\%$ & $n .^{\circ}$ de respuestas & $\%$ \\
\hline Interacciones participantes & 3 & 37,5 & 3 & 50,0 & 0 & 0,0 \\
\hline Integración de áreas & 3 & 37,5 & 3 & 50,0 & 0 & 0,0 \\
\hline Construcción del conocimiento & 2 & 25,0 & 2 & 33,3 & 0 & 0,0 \\
\hline Aplicación en las prácticas de aula & 2 & 25,0 & 2 & 33,3 & 0 & 0,0 \\
\hline Apoyo virtual & 2 & 25,0 & 2 & 33,3 & 0 & 0,0 \\
\hline Fortalecimiento de la escritura académica & 1 & 12,5 & 0 & 0,0 & 1 & 50,0 \\
\hline
\end{tabular}

Fuente: elaboración propia. 
Boyd y Mohamad (2011) plantean la importancia de que el CE esté asociado con programas o estrategias institucionales, desde donde se pueda aportar a la escritura. Desde el liderazgo institucional, en el caso 1, el 50\% de los tutores del CED acompañó la escritura de artículos, por parte de los estudiantes, para una publicación institucional; esta actividad se realizó desde la tutoría académica. En el caso 1 el 33.3\% y en el caso 2 el 50\% de los tutores desarrollaron orientaciones a proyectos técnicos y transversales; para ello, se implementaron dos acciones: las tutorías académicas y el apoyo a otros profesores.

\section{Estrategias de promoción de los servicios}

Los coordinadores de cada uno de los CED tenían bajo su responsabilidad promover y divulgar los servicios. No tenían que hacerlo solos, pero sí liderar las actividades y ser el enlace entre las propuestas que se dieran entre los integrantes del CED y los directivos de la institución. Por ejemplo, en el caso 1, el coordinador académico redactó una carta de motivación dirigida a los profesores de la institución educativa para invitarlos a hacer uso del CED en sus clases y que convocaran a los estudiantes a hacer uso de las tutorías académicas. En el caso 2, la coordinadora académica durante una reunión de profesores realizó una presentación del CED y les explicó las posibilidades de uso en la cátedra de desarrollo del pensamiento.

Utne (2016) considera que los profesores deben recomendar en sus clases los servicios del CED. Además, de manera individual, deben orientar algunos estudiantes para que soliciten las tutorías académicas. Los profesores de las instituciones educativas fueron comunicadores directos de los servicios del CED. Desde la investigación y con apoyo de los coordinadores, a los profesores se les socializó el alcance del CED, sus propósitos, los retos que implicaba su implementación y se les invitó a participar y promover entre los estudiantes las actividades que convocaba el CED. Los recursos ofrecidos por el CED no beneficiarían únicamente a los estudiantes, debido a que fueron también un apoyo para los profesores en cuanto a que con ellos se proporcionaba ayuda a los estudiantes y se les brindaba talleres y asesoría académica, para que mejoraran la enseñanza y el aprendizaje de la escritura dentro de las áreas.

Los estudiantes de ambos casos, en una gran mayoría, manifestaron que se enteraron de los servicios que ofrece el CED por los profesores y directivos (caso $1,93.5 \%$ y caso $2,71.4 \%$ ); estos fueron fundamentales al momento de promocionar el CED en la comunidad educativa, debido a que eran los que tenían el contacto directo con los estudiantes en el aula de clases (ver Tabla 13). Además, los profesores tenían la posibilidad de remitir a los estudiantes a que utilizaran los recursos que se encontraban en el CED o participar de uno de los servicios. En el caso 1, el 12.9\% estudiantes expresó haberse enterado por la página web institucional, lo que evidencia la importancia de integrar el CED a los medios de comunicación institucionales. En el caso 2, el 42.9\% de los estudiantes dijo haberse enterado por otros estudiantes, lo que reflejó que el CED hizo parte de las conversaciones de compañeros de clases.

Tabla 13. Canales de comunicación del ced reconocidas por los estudiantes

\begin{tabular}{|c|c|c|c|c|c|c|}
\hline & \multicolumn{2}{|l|}{ Total } & \multicolumn{2}{|l|}{$\begin{array}{c}\text { Caso 1: } \\
\text { IE Pública }\end{array}$} & \multicolumn{2}{|l|}{$\begin{array}{l}\text { Caso 2: } \\
\text { IE Privada }\end{array}$} \\
\hline & $n .^{\circ}$ de respuestas & $\%$ & $n .^{\circ}$ de respuestas & $\%$ & $n .^{\circ}$ de respuestas & $\%$ \\
\hline Profesores & 34 & 89,5 & 29 & 93,5 & 5 & 71,4 \\
\hline Página web institucional & 4 & 10,5 & 4 & 12,9 & 0 & 0,0 \\
\hline Otros estudiantes & 3 & 7,9 & 0 & 0,0 & 3 & 42,9 \\
\hline Directivos & 2 & 5,3 & 1 & 3,2 & 1 & 14,3 \\
\hline
\end{tabular}

Fuente: elaboración propia. 
Faigley (1998) propone que los CE deben educar permanentemente a las comunidades educativas alrededor de lo que hacen. Un amplio porcentaje de profesores de ambos casos expresaron que ellos socializaron los servicios y recursos del CED desde el trabajo que se realizaba en sus clases (caso 1, $66.7 \%$ y caso $2,50 \%$ ), es más, los recursos de la biblioteca virtual fueron incorporados en varias dinámicas de enseñanza (ver Tabla 14). Además, la mitad de profesores en ambos casos reconoció que los espacios digitales con que cuenta la institución fueron importantes para promover el CED en la comunidad educativa (caso $1,50 \%$ y caso 2 , 50\%). Entre estos espacios se encuentran: página web, plataformas educativas, blog de áreas, correo electrónico institucional o personal. Otro espacio de promoción fue el de las carteleras institucionales (caso $1,33.3 \%$ y caso $2,50 \%$ ) donde tradicionalmente se realizan estrategias informativas y motivacionales. En el caso 1, se diseñó y desarrolló una orientación de grupo, para que los profesores contaran y orientaran a los estudiantes sobre los servicios del CED destinados para ellos.

Igualmente, la mitad de los profesores encuestados en ambos casos reconoce los comunicados institucionales como una estrategia para divulgar los servicios del Ced (caso 1, 50\% y caso 2, 50\%). Por ejemplo, en el caso 1 se diseñó un plegable para que los estudiantes y padres de familia conocieran la forma de ingresar al CED y los servicios y recursos que se encontraban en este espacio. En el caso 2, se publicó un artículo en el periódico institucional contando a la comunidad educativa lo que se venía realizando desde el CED y cómo se beneficiaban los estudiantes.

Formar a la comunidad educativa para que comprenda el papel del CED en la institución en el fortalecimiento de la escritura académica fue necesario para que los estudiantes hicieran uso del CED. Las estrategias de promoción y divulgación, en ambas instituciones educativas, fueron variadas, entre las cuales se realizaron: cartelera informativa, afiches publicitarios, plegables, carta a profesores, circulares a padres de familia, uso de la página web para la divulgación, reuniones informativas padres de familia y estudiantes. Además, en el caso 2, se realizó una nota sobre el CED en el periódico escolar.

\section{Integración curricular}

El CED de cada institución aportó a la comprensión y desarrollos de la escritura a través de las asignaturas y áreas del currículo escolar. Aunque cada institución educativa consideraba importante la lectura y escritura en todas las áreas, las propuestas que se desarrollaban correspondían a las áreas del lenguaje. Con el CED se continuó con la idea de la responsabilidad de todas las áreas en la escritura. Además, los profesores y estudiantes comenzaron a preguntarse cómo trabajar la escritura en cada área y cuáles serían sus aportes al aprendizaje. En el caso 2, el CED aportó a la cátedra de desarrollo del pensamiento que la institución ha implementado desde hace más de cuatro años, no solo desde

Tabla 14. Estrategias de comunicación del ced reconocidas por los profesores

\begin{tabular}{|c|c|c|c|c|c|c|}
\hline & \multicolumn{2}{|l|}{ Total } & \multicolumn{2}{|l|}{$\begin{array}{l}\text { Caso 1: } \\
\text { IE Pública }\end{array}$} & \multicolumn{2}{|l|}{$\begin{array}{l}\text { Caso 2: } \\
\text { IE Privada }\end{array}$} \\
\hline & n. ${ }^{\circ}$ de respuestas & $\%$ & n. ${ }^{\circ}$ de respuestas & $\%$ & $n .^{\circ}$ de respuestas & $\%$ \\
\hline Socialización en las clases & 5 & 62,5 & 4 & 66,7 & 1 & 50,0 \\
\hline Recursos digitales & 4 & 50,0 & 3 & 50,0 & 1 & 50,0 \\
\hline Comunicados & 4 & 50,0 & 3 & 50,0 & 1 & 50,0 \\
\hline Reuniones de profesores & 3 & 37,5 & 3 & 50,0 & 0 & 0,0 \\
\hline Carteleras institucionales & 3 & 37,5 & 2 & 33,3 & 1 & 50,0 \\
\hline Orientación de grupo & 2 & 25,0 & 2 & 33,3 & 0 & 0,0 \\
\hline
\end{tabular}

Fuente: elaboración propia. 
los recursos que les ofrecía el CED, también desde los aportes teóricos y prácticos para el trabajo de la escritura, por parte de los profesores.

La incorporación del CED en el diseño curricular de la institución se dio en dos direcciones: lo que el CED le fue otorgando a las instituciones y las adaptaciones que fueron realizando las instituciones para responder a los retos de tener un CED (ver Tabla 15). En ambos casos, algunos de los talleres que se proponían en el CED fueron incorporados a las dinámicas de las clases, por ejemplo en el caso 1, en el área de Tecnología e Informática, los estudiantes del grado décimo trabajaron el taller sobre escritura de presentaciones para la expresión oral y en el área de Filosofía, el informe de lectura; en el caso 2, en la asignatura de Lengua Castellana, los estudiantes de grado décimo trabajaron el taller de la reseña crítica y en el área de Tecnología e Informática, el resumen.

Faigley (1998) afirma que un CE debe responder a las realidades institucionales, identificando los problemas en materia de escritura y aprendizaje, y actuando sobre ellos. En ambos casos, los estudiantes identificaron que los servicios del CED se utilizaron en las actividades institucionales para el apoyo en las actividades académicas en las áreas del currículo escolar (caso 1, 54.8\% y caso 2,
42.9\%). Por ejemplo, los profesores socializaron una de las guías para que los estudiantes hicieran un comentario sobre un tema específico en el blog del área. Igualmente, un amplio porcentaje de los estudiantes reconoce que el CED permite el aprendizaje autónomo (caso 1, 35.5\% y caso 2, 57.1\%). Por ello, el uso se dio accediendo a los recursos virtuales que necesitaron, los exploraron y los aplicaron en las tareas de escritura (ver Tabla 16).

El CED, también, realizó algunos ajustes durante su proceso de implementación. Aunque inicialmente las actividades estaban pensadas para desarrollarse de manera virtual y en la educación media, se fueron haciendo los ajustes pertinentes para que los profesores de los diferentes grados pudieran utilizarlos. Así, en el caso 1, los profesores de la educación básica secundaria utilizaban los recursos de la biblioteca virtual; y el profesor Tecnología e Informática de los grados séptimo y octavo utilizó la guía de escritura de comentarios en un blog para trabajarla en las clases, previamente hubo una asesoría y acompañamiento de un profesor/tutor del CED para adaptarlo a las competencias del área. En el caso 2, una profesora de Lengua Castellana del grado octavo utilizó la guía de escritura de argumentos para trabajarla en sus clases; para ello, la coordinadora del ceD le dio

Tabla 15. Usos del CED por parte de los profesores

\begin{tabular}{|c|c|c|c|c|c|c|}
\hline & \multicolumn{2}{|l|}{ Total } & \multicolumn{2}{|l|}{$\begin{array}{l}\text { Caso 1: } \\
\text { IE Pública }\end{array}$} & \multicolumn{2}{|l|}{$\begin{array}{l}\text { Caso 2: } \\
\text { IE Privada }\end{array}$} \\
\hline & n. ${ }^{\circ}$ de respuestas & $\%$ & $\mathrm{n} .^{\circ}$ de respuestas & $\%$ & $\mathrm{n} .^{\circ}$ de respuestas & $\%$ \\
\hline Complemento trabajo de clases & 5 & 62,5 & 3 & 50,0 & 2 & 100,0 \\
\hline Orientación escritura académica & 4 & 50,0 & 4 & 66,7 & 0 & 0,0 \\
\hline Planes de mejoramiento académico & 1 & 12,5 & 1 & 16,7 & 0 & 0,0 \\
\hline
\end{tabular}

Fuente: elaboración propia.

Tabla 16. Usos del CED en la institución según los estudiantes

\begin{tabular}{|c|c|c|c|c|c|c|}
\hline & \multicolumn{2}{|l|}{ Total } & \multicolumn{2}{|l|}{$\begin{array}{l}\text { Caso 1: } \\
\text { IE Pública }\end{array}$} & \multicolumn{2}{|l|}{$\begin{array}{l}\text { Caso 2: } \\
\text { IE Privada }\end{array}$} \\
\hline & $n .^{\circ}$ de respuestas & $\%$ & $\mathbf{n}{ }^{\circ}$ de respuestas & $\%$ & $\mathrm{n} .^{\circ}$ de respuestas & $\%$ \\
\hline Apoyo a las áreas & 20 & 52,6 & 17 & 54,8 & 3 & 42,9 \\
\hline Autonomía del estudiante & 15 & 39,5 & 11 & 35,5 & 4 & 57,1 \\
\hline
\end{tabular}

Fuente: elaboración propia. 
algunas sugerencias y se realizaron algunos ajustes a la guía para poder ser trabajada en el grado escolar.

Las dinámicas del CED generaron que se hicieran algunos ajustes en los planes de área. Por ejemplo, en el caso 2, la profesora del área de Tecnología e Informática actualizó el plan de área para incorporar acciones que estuvieran asociadas al CED, como el uso de recursos virtuales para la escritura, el uso de la biblioteca escolar para la alfabetización digital, el acompañamiento de la escritura en los proyectos tecnológicos. Vale anotar que el CED, desde un comienzo, se pensó para responder a la estructura curricular de las áreas de ambas instituciones educativas; por ello, algunos de los talleres fueron exclusivos para las instituciones haciendo énfasis en los propósitos de aprendizaje planteados en cada una. Igualmente, algunos recursos de la biblioteca virtual se diseñaron e incorporaron respondiendo a intereses expresados en el cuestionario de indagación por los profesores y estudiantes. Stueart (2012) considera que los profesores de las diferentes áreas deben estar en capacidad de usar el CE para mejorar sus prácticas de enseñanza de escritura.

Las acciones del CED, también, aportaron a los procesos de evaluación de los aprendizajes de los estudiantes. En el caso 1, en el Sistema Institucional de Evaluación se plantea que el $20 \%$ de la calificación final debe corresponder a una tarea escolar; los profesores de Lengua Castellana, Educación Física, Tecnología e Informática, Emprendimiento, Ciencias Económicas y Políticas, Filosofía, Multimedia, Programación, en algunos de los periodos académicos utilizaron uno de los talleres o recursos de la biblioteca del CED para asignar la tarea escolar y adelantar el proceso de evaluación. Además, para el módulo de profesores se diseñó un taller de escritura de pruebas tipo Saber, orientado a los procesos de evaluación dentro de las áreas.

McAndrew y Reigstad (2001) y Hewett (2010) consideran protagónico el papel de los tutores en un CE. El 100\% de los tutores de ambos casos identifican que la comunidad educativa reconoció el CED como un espacio para el apoyo institucional. En el caso 1, el $83.3 \%$ y en el caso 2 el $100 \%$ de los tutores plantearon que ese reconocimiento se observa en la iniciativa de los estudiantes para solicitar, acceder y asistir a los servicios; los estudiantes se mostraron familiarizados con el CED y, aunque no lo utilizaran con frecuencia, era notoria la disposición para intervenir y aportar a los ejercicios de escritura de los compañeros, en el espacio digital.

En el caso 1, el 33.3\% de los tutores planteó que otra forma de ver la apropiación del CED por parte de la comunidad educativa fue su uso en las actividades académicas, donde los profesores llevaron a las clases materiales virtuales tomados del CED, o planearon sus clases desde los recursos que este ofrecía. El 16.7\% de los tutores identificó que las estrategias de divulgación realizadas para el reconocimiento del CED, a través de la página, comunicados y circulares, mostraron cómo diferentes actores de la comunidad aportaron a la consolidación del CED en la institución (ver Tabla 17).

\section{Conclusiones}

Un CED en la educación básica y media proporciona un valor diferenciador de la institución entre otros espacios educativos de la ciudad. Se genera

Tabla 17. Reconocimiento del CED según los tutores

\begin{tabular}{|c|c|c|c|c|c|c|}
\hline & \multicolumn{2}{|l|}{ Total } & \multicolumn{2}{|l|}{$\begin{array}{c}\text { Caso 1: } \\
\text { IE Pública }\end{array}$} & \multicolumn{2}{|l|}{$\begin{array}{l}\text { Caso 2: } \\
\text { IE Privada }\end{array}$} \\
\hline & $\mathbf{n} .^{\circ}$ de respuestas & $\%$ & n. ${ }^{\circ}$ de respuestas & $\%$ & n. ${ }^{\circ}$ de respuestas & $\%$ \\
\hline Iniciativa del estudiante & 7 & 87,5 & 5 & 83,3 & 2 & 100,0 \\
\hline Uso del CED en las clases & 2 & 25,0 & 2 & 33,3 & 0 & 0,0 \\
\hline Divulgación institucional & 1 & 12,5 & 1 & 16,7 & 0 & 0,0 \\
\hline
\end{tabular}

Fuente: elaboración propia. 
una proyección, donde el CED se reconoce como una estrategia institucional para potenciar la escritura en los estudiantes. Esta diferencia aporta a la identidad, al reconocimiento de los estudiantes y padres de familia como un valor agregado de los servicios de la institución, a construir lazos de colaboración con otras instituciones que se encuentren interesadas en diseñar e implementar un CE. Sin embargo, para lograr ese valor distintivo se requiere un proceso riguroso en el diseño, la operación, seguimiento y evaluación del CED desde la realidad, respondiendo a los propósitos formativos de la institución planteados en su PEI.

En la relación PEI y CED, favorecer desarrollos alrededor de la innovación educativa implicaba la comprensión de la historia institucional, sus territorios y su realidad actual. Esa comprensión permitió que el CED se estableciera dentro de las instituciones y aportara al desarrollo del concepto de innovación educativa, no por el hecho de utilizar las TIC, sino por lograr abordar el problema de la escritura académica desde otra perspectiva que, aunque partía de modelos anglosajones, se adaptó a los objetivos institucionales para la educación media. García-Peñalvo (2015) afirma que la innovación educativa supone la unión de sinergias entre crear algo nuevo, el proceso y la mejora del proceso, en un contexto donde se desarrolla la innovación. En el diseño e implementación del CED se consideró que, al ser una experiencia nueva en ambas instituciones, era importante que las poblaciones conocieran los procesos a desarrollar y participaran de su construcción.

El CED aportó a los procesos de alfabetización digital en las instituciones educativas. Avello et al. (2013) plantean que la alfabetización digital abarca las competencias que la persona necesita para desempeñarse en la sociedad de la información y el conocimiento. El CED aportó al desarrollo de esas competencias en los estudiantes. Un ejemplo de ello es que cuando un estudiante se aproximaba a la escritura de un texto académico utilizando los recursos de la biblioteca virtual requería acceder, buscar, analizar, seleccionar, evaluar la información que se le ofrecía para luego leerla, interpretarla, aplicarla con el propósito de construir o mejorar su proceso de escritura. En el CED se consideró que la alfabetización digital supera los procesos instrumentales del uso de las TIC, implica comprender las posibilidades de uso de las TiC para el desempeño en la sociedad.

Que el personal de la institución tuviera un acercamiento o experiencias previas sobre el uso de las TIC en la escuela aportó a comprender los retos y oportunidades del proceso de implementar un CED en las instituciones. En un CED el uso de las TIC supera la enseñanza del uso de herramientas, implica el fortalecimiento de los procesos de alfabetización digital desde las áreas y asignaturas del currículo escolar, donde se involucren los directivos, profesores, estudiantes y padres de familia. Revuelta Domínguez y Arriazu Muñoz (2015) afirman que las instituciones educativas deben ser organizaciones estratégicas que marcan la política educativa de un país y asuman sus responsabilidades en el proceso de incorporación de las TIC, partiendo de las implicaciones e importancia de los profesores en el proceso de integración.

Un CED debe estar en diálogo con el Modelo Pedagógico Institucional. La propuesta de los CE alrededor del mundo está orientada a un aprendizaje colaborativo. Por lo tanto, cuando una institución educativa toma la decisión de operar un CED, su Modelo Pedagógico mantendrá una relación social y constructivista del aprendizaje, favoreciendo la formación colaborativa y autónoma del estudiante. Pensar un CED dentro de una propuesta pedagógica que no considere al otro o donde la enseñanza de la lectura y la escritura sean un problema de las áreas del lenguaje, no tendrá una base sólida para su desarrollo y sostenibilidad. En el caso 1, la institución educativa pública, el modelo pedagógico fue "Holístico Transformador" y dentro de su descripción se privilegia el aprendizaje colaborativo, el uso de las TIC para el aprendizaje, la investigación escolar y el desarrollo de las habilidades comunicativas. En el caso 2, la institución educativa privada, la propuesta pedagógica es "Desarrollista", dentro de sus postulados se destacan los ejes de mediación, aprendizaje significativo, investigación y liderazgo.

Diseñar e implementar estrategias de promoción y divulgación del CED tenía como propósito dar a conocer a la comunidad educativa los 
servicios y alcance del CED dentro de la institución educativa. Para ello, cada institución educativa fue autónoma y las desarrollaba desde los recursos disponibles y las autorizaciones pertinentes de los directivos. Utne (2016) plantea que el apoyo de los administradores de las instituciones educativas y los profesores repercutirá en el éxito del CE. Por ello, las estrategias de divulgación no solo estaban enfocadas en los estudiantes, también se dirigieron hacia los directivos, profesores y padres de familia.

Para que los CED se mantuvieran en las instituciones era importante crear estrategias de seguimiento y evaluación, que reflejaran los desarrollos que se venían dando en los CED y tomar decisión en el mejoramiento de los servicios prestados a la comunidad educativa. En ambos casos, las estrategias de seguimiento y evaluación estuvieron dadas desde el registro del número de participantes por servicios y la evaluación de los servicios que ofrecía el CED por parte de los usuarios. Ackley (1989) y Utne (2016) sugieren tener registro de las sesiones de tutoría con el propósito de que los directores del CE puedan tomar decisiones. Tener indicadores para el seguimiento y evaluación de las dinámicas del CED se convierte una estrategia para informar a las directivas de la institución de los desarrollos y avances que se vayan dando en el Centro. Entre los posibles indicadores se plantean la relación entre CED y el rendimiento académico de los estudiantes, la deserción escolar entre los usuarios del CED, el ingreso a la educación superior de los estudiantes que hacían uso del CED. Lo anterior concuerda con los postulados de Bell y Frost (2012) sobre la importancia de diseñar herramientas de autoevaluación en los $\mathrm{CE}$, con los propósitos de hacer seguimiento y documentar lo que se realiza para beneficio de las instituciones educativas.

Los padres de familia podrían actuar como un grupo de voluntariado para apoyar acciones de promoción, acompañamiento y logística dentro del CED; además, aquellos padres con formación profesional serían invitados a formar parte del grupo de tutores. La decisión de operar un CED en la educación media implica renovar las relaciones entre la escuela y la familia. En la educación básica y media los padres de familia tienen un valor importante en la formación de los estudiantes, y en un CED no es la excepción. En el proceso de reconocimiento del CED como parte del PEI, las instituciones educativas crearon mecanismos de comunicación y acercamiento a los profesores, estudiantes y padres de familia. Estos repercutieron positivamente en el posicionamiento y uso del CED.

Una limitación de la investigación fue la atención a poblaciones diversas, como indígenas y estudiantes con necesidades educativas especiales. Durante la implementación se presentaron casos de estudiantes con problemas cognitivos que solicitaron tutorías académicas; sin embargo, no se contaba con el profesional de apoyo que acompañara este tipo de procesos, debido a las características específicas que implica el apoyo pedagógico y didáctico a estos estudiantes. Este aspecto se convierte en una oportunidad de mejora: pensar la forma de atender a poblaciones diversas, desde el CED.

Al comenzar la investigación no se consideró el apoyo técnico que se requería en el interior de las instituciones para los estudiantes, debido a que se suponía que ellos ya dominaban el componente técnico de las plataformas educativas, lo que generó que los profesores de Tecnología e Informática de los grados décimo y undécimo asumieran este acompañamiento dentro de las instituciones. Por lo anterior, como sugerencia para la apertura de un CED, se propone considerar la creación de una mesa de ayuda técnica, que sea responsable de orientar a los usuarios en la parte instrumental, actualizar la información, servicios de la plataforma y asignación de recursos tecnológicos para la prestación de los servicios presenciales con apoyo de las TIC que se ofrezcan.

Como recomendaciones para futuras investigaciones sobre los CED en la escuela, se plantea pensar el rol del tutor par, considerando los mecanismos de selección, rutas de formación, acompañamiento y evaluación del proceso, reconocimiento del tiempo que dedica a la atención de los estudiantes, debido al potencial que tiene la incorporación de tutores pares para la consolidación del CED en la institución educativa. Sin embargo, esto implicaría que el coordinador del CED genere un ambiente de trabajo donde los tutores puedan desempeñar determinadas funciones con su acompañamiento, 
pero con un nivel de autonomía que les permita orientar los servicios bajo unos principios administrativos y pedagógicos, y desde su experiencia.

Un servicio que se podría ofertar desde el CED es la promoción y desarrollo de eventos académicos, como videoconferencias, foros virtuales, para estudiantes y profesores, alrededor de la lectura, escritura y oralidad. Además, el CED podría apoyar la transversalización de una política sobre el trabajo de la escritura académica dentro de las áreas, teniendo presente que los servicios que se ofrezcan respondan a la capacidad instalada, objetivos formativos y recursos económicos. Además, se deben tener presentes los principios pedagógicos y didácticos que orientan al CED en la institución. No se trata de crear servicios aleatoriamente, es fundamental que estos se incorporen a la realidad institucional y, previamente, hayan sido socializados entre directivas, profesores y estudiantes.

Un CED en la educación media reafirma el propósito pedagógico de formar mejores escritores, lo que repercutirá en oportunidades para que los estudiantes enfrenten, con herramientas cognitivas y sociales, asuntos académicos como la presentación de pruebas de lectura y escritura, exámenes de selección, sus primeros semestres en la educación superior y actividades comunicativas que se requieren para ser ciudadanos activos. Un CED ha de mantener ese carácter articulador de los programas y proyectos institucionales para el fortalecimiento de la escritura en los estudiantes, sin embargo, cada institución educativa determina las formas de diseño, operación y seguimiento, respondiendo a sus realidades administrativas, curriculares y sociales.

\section{Referencias}

Ackley, E. (1989). Training Peer Tutors for the Secondary School Writing Center. En Pamela Farrell (Ed.), The High School Writing Center: Establishing and Maintaining One (pp. 65-72). Urbana, Illinois: National Council of Teachers of English. Recuperado de http://wac. colostate.edu/books/hswc/book.pdf

Aponte, S. A. y Cruz, L. C. (2015). Más allá de las líneas de la revista escolar (Tesis de Maestría). Facultad de Educación, Universidad Santo Tomás, Bogotá. Recuperado de http://repository.usta.edu.co/bitstream/
handle/11634/549/Mas\%20alla\%20de\%20las\%20lineas $\% 20$ de $\% 201 a \% 20$ revista\%20escolar.pdf?sequen$\mathrm{ce}=1$ \&isAllowed $=\mathrm{y}$

Avello, R., López, R., Cañedo, M., Álvarez, H., Granados, J. y Obando, F. (2013). Evolución de la alfabetización digital: nuevos conceptos y nuevas alfabetizaciones. MediSur, 11(4), 450-457. Recuperado de http:// scielo.sld.cu/scielo.php?script $=$ sci_arttext\&pi$\mathrm{d}=$ S1727-897X2013000400009

Ávila, C. (2009) El periódico escolar virtual, una estrategia de integración para el aprendizaje autónomo desde las competenciaslecto-escritoras (Tesis de especialización). Escuela de Posgrados, Universidad Nacional Abierta y a Distancia (UNAD) Recuperado de http://repository. unad.edu.co/bitstream/10596/2441/1/02-2014-16.pdf

Bell, D. C. y Frost, A. (2012). Critical Inquiry and Writing Centers: A Methodology of Assessment. Learning Assistance Review, 17(1), 15-26. Recuperado de http://files.eric.ed.gov/fulltext/EJ973400.pdf

Boyd, J. y Mohamad, M. (2011). Different Maximum Values in Writing Centers. Academic Exchange Quarterly, 15(4), 8. Recuperado de http://www.rapidintellect. com/AEQweb/cho4941.htm

Calle-Álvarez, G. Y. (2019). Estrategias de implementación de un centro de escritura digital en la educación media. Trilogía Ciencia Tecnología Sociedad, 11(21), 311336. DoI: https://doi.org/10.22430/21457778.1297

Calle-Arango, L. (2020). Centros y programas de escritura en las IEs colombianas. Magis, Revista Internacional de Investigación en Educación, 12(25), 77-92. Dor: https:// doi.org/10.11144/Javeriana.m12-25.cpei

Carrasco, A. (2019). De la escuela a la universidad: las prácticas de lectura y escritura en el último año de la secundaria. Letras, (8). Recuperado de https://www.perio.unlp.edu.ar/ojs/index.php/letras/article/view/5780

Congreso de Colombia. (8 de febrero de 1994) Ley General de Educación [Ley 115 de 1994]. Do: 41.214. Recuperado de http://www.mineducacion.gov.co/1621/articles-85906_archivo_pdf.pdf

Eckard, S. y Stroudsburg, E. (2013). Facebook as a Tool for Both Writing Centers and Academic Classrooms. EAPSU Online: A Journal of Critical and Creative Work, 102.

Eleftheriou, M. (2011). An exploratory study of a middle eastern writing center: The perceptions of tutors and tutees (Tesis doctoral). Universidad de Leicester. Recuperado de https://pdfs.semanticscholar.org/717c/ 36628flaafcf57f8484aa017825e52768c8b.pdf

Faigley, L. (1998). Writing centers in times of whitewater. The Writing Center Journal, 19(1), 7-18. 
García, F. (2002). El cuestionario: recomendaciones metodológicas para el diseño de cuestionarios. México: Editorial Limusa.

García-Peñalvo, F. J. (2015). Mapa de tendencias en Innovación Educativa. Teoría de la educación, educación y cultura en la sociedad de la información, 16(4), 6-23. Recuperado de https://revistas.usal.es/index.php/eks/ article/view/eks2015164623/13764. DOI: https://doi. org/10.14201/eks2015164623

Griswold, G. (2003). Writing centers: The student retention connection. Academic Exchange Quarterly, 7(4), 277-282.

Hewett, B. L. (2010). The online writing conference: A guide for teachers and tutors. Portsmouth, NH: Boynton/ Cook.

Holtz, E. V. (2014), Mode, Method, and Medium: The Affordance of Online Tutorials in the Writing Center. Honors Scholar Theses, Universidad de Connecticut, Estados Unidos. Recuperado http://digitalcommons. uconn.edu/cgi/viewcontent.cgi?article $=1352 \&$ contex$\mathrm{t}=$ srhonors_theses

McAndrew, D. A., y Reigstad, T. J. (2001). Tutoring writing: A practical guide for conferences. Portsmouth NH: Heinemann-Boynton/Cook

Meuller, G. (2006). A call to action: Embracing the need for high school writing centers. The Writing Lab Newsletter, (30), 10-11.

Ministerio de Educación Nacional de Colombia. (2006). Estándares Básicos de Competencias en Lenguaje, Matemáticas, Ciencias y Ciudadanas. Guía sobre lo que los estudiantes deben saber y saber hacer con lo que aprenden. Imprenta Nacional de Colombia. Recuperado de http://www.mineducacion.gov.co/1621/articles-340021_recurso_1.pdf

Rodríguez, E., Muñoz, J. y Ángel, D. M. Á. (2015). Desarrollo producción textual en estudiantes educación media técnica. Propuesta: taller de escritores. Amazonia Investiga, 4(6). Recuperado de http://www.udla. edu.co/revistas/index.php/amazonia-investiga/article/ viewFile/356/pdf
Simpson, J. (1991). The role of writing centers in student retention. In R. Wallace y J. Simpson (Eds.), The Writing Center: New Directions (pp. 102-109). New York: Garland.

Stueart, K. C. (2012). A Proposal for a Writing Center and a Peer Tutor Training Course at Fayetteville High School (Tesis de Maestría). Universidad de Arkansas, Estados Unidos. Recuperado de http://scholarworks.uark.edu/ cgi/viewcontent.cgi? article $=1284 \&$ context $=$ etd

Tesch, R. (1990). Qualitative analysis: Analysis types and software tools. London: Falmer.

Torres, F. J. (2013). Kukuguagua: propuesta de un programa de lectura digital desde la biblioteca escolar del Centro Educativo Palermo Sur de la localidad Rafael Uribe (Tesis de pregrado). Facultad de Comunicación y Lenguaje, Pontificia Universidad Javeriana, Bogotá. Recuperado de https://repository.javeriana.edu.co/ bitstream/handle/10554/15196/TorresSolerFrancyJas$\min 2013$.pdf? sequence $=1$ \&isAllowed $=\mathrm{y}$

Utne, K. L., (2016). A Proposal for A Writing Center at Lafayette High School (Tesis de pregrado). Universidad de James Madison. Recuperado de http://commons.lib. jmu.edu/honors201019/204/

Villasevil, F. J. (2016). Influencia de los multimedia (tic-tac) en el proceso enseñanza/aprendizaje (Tesis Doctoral). Universitat Politècnica de Catalunya, España. Recuperado de https://dialnet.unirioja.es/servlet/tesis?co$\operatorname{digo}=133293$

Werner, C. L. (2013). Constructing Student Learning through Faculty Development: Writing Experts, Writing Centers, and Faculty Resources. The CEA Forum, College English Association, 42(2), 79-92. Recuperado de: http://files.eric.ed.gov/fulltext/EJ1018288.pdf

Yacuzzi, E. (2005). El estudio de caso como metodología de investigación: teoría, mecanismos causales, validación. Serie Documentos de Trabajo, Universidad del CEMA. Área de negocios, (296). Recuperado de https://www. econstor.eu/bitstream/10419/84390/1/496805126.pdf

Yin, R. (1994). Case study research: Design and methods. Beverly Hills, CA: Sage Publishing. 
